\title{
Are Cluster Dwarfs Recycled Galaxies?
}

\author{
Christopher J. Conselice \\ California Institute of Technology, Mail Code 105-24 \\ Pasadena CA 91125, USA
}

\begin{abstract}
Although cluster dwarf galaxies are often neglected due to their faintness, recent observations demonstrate they may be critical for understanding the physical processes behind galaxy formation. Dwarfs are the most common galaxy type and are particularly abundant in clusters. Recent observational results suggest that dwarfs in dense environments do not all form early in the universe, as expected in hierarchical structure formation models. Many of these systems appear to be younger and more metal rich than dwarfs in lower density areas, suggesting they are possibly created by a tidal process. Several general galaxy cluster observations, including steep luminosity functions and the origin of intracluster light, are natural outcomes of these processes.
\end{abstract}

\section{Introduction}

The most common galaxy in the universe are dwarf galaxies. These systems are typically faint $\left(\mathrm{M}_{\mathrm{B}}>-18\right)$, and have a low surface brightness $(\mu>23$ mag $\left.\operatorname{arcsec}^{2}\right)$. Since dwarfs are so common, they are in every sense normal galaxies. The most common type among dwarfs are dwarf ellipticals/spheroids, which dominate the number density of galaxy clusters down to $\mathrm{M}_{\mathrm{B}}=-11$ (Ferguson \& Bingelli 1994; Trentham et al. 2002). Based on studies of luminosity functions in clusters, there are also more dwarf ellipticals per giant in denser regions than in the field. This means, in the most simplest terms, that clusters of galaxies cannot form through simple mergers of galaxy groups. Some dwarf systems must form within the cluster environment. The nature of this overdensity may be the result of initial conditions, or the result of 'non-standard' galaxy formation. That is, dwarfs may have formed after the cluster was in place. There is now ample evidence for this, the implications of which can explain several galaxy cluster phenomena.

The first clue that dwarfs are not produced through standard methodologies came from studies that showed dwarfs are generally found in abundance in dense areas such as nearby clusters. In fact, in standard CDM scenarios dwarfs should be more common in lower density environments, but we see the direct opposite (Trentham et al. 2002). Dwarfs within dense environments also have a broader distribution, both spatially and in terms of their radial velocities, than the giant ellipticals, similar to the pattern of infalling spirals. This is an indication that both are more recent additions to clusters (Conselice et al. 2001). Internal dynamic evidence also suggests that at least some dwarfs are rotating (e.g., 
Geha et al. 2003), a feature not seen in Local Group dEs. Finally, the stellar populations of some faint $\mathrm{M}_{\mathrm{B}}>-15$ dwarf galaxies appear to be metal rich (near solar), implying that the dwarf population itself is inhomogeneous and may have multiple origins. We discuss several scenarios which have been proposed to explain these observations and argue that one method which can reproduce these trends involves the formation of dwarfs from already existing galaxies through a tidal effect.

\section{Dwarf Galaxy Properties}

The two places where dwarfs have been studied in detail are in nearby rich clusters, such as Virgo, Fornax and Coma, and in the Local Group. Most of the data we discuss therefore comes from these sources. In particular a significant fraction of the data presented in this article comes from the papers by Conselice, Gallagher \& Wyse $(2001,2002,2003)$ and Conselice et al. (2003b). We list below our current understanding of dwarf properties in terms of various observational quantities.

Number Densities: The luminosity functions (LF) in all galaxy environments are dominated by dwarf galaxies. The overdensity of dwarfs in clusters is about 5-10 times that in groups. Another way to quantify this is through the faint end slope of the LF, $\alpha$. The value of $\alpha$ in rich clusters, such as Virgo is around $\alpha=$ -1.4 (Sandage et al. 1985; Conselice et al. 2002), with some results suggesting even steeper LF with $\alpha=-1.6$ (Sabatini et al. 2003). This is steeper than field values, such as in the Local Group $(\alpha=-1.1$; van den Bergh 2000), yet flatter than the value predicted by CDM for all environments $(\alpha=-2)$. Environment clearly affects the way these systems are produced, which is generally not a prediction in CDM models (cf. Tully et al. 2002).

Spatial Positions: While Local Group dwarf galaxies, particularly dwarf ellipticals, are strongly clustered around giant galaxies in the Local Group (e.g., van den Bergh 2000), the opposite is found for low-mass galaxies in clusters, where most are neither clustered around, nor distributed globally similar to, giant elliptical galaxies (Conselice et al. 2001). Both dwarf ellipticals and irregulars also have a broader distribution in clusters, that is they are not clustered towards the center, but are spread throughout (e.g., Conselice et al. 2001).

Radial Velocities: The radial velocities of low-mass cluster galaxies, including S0s, spirals, dwarf irregulars and dwarf ellipticals have a wider distribution than the ellipticals (see Figure 1a). For example, Virgo cluster elliptical galaxies have a narrow Gaussian velocity distribution, with $\sigma=462 \mathrm{~km} \mathrm{~s}^{-1}$, concentrated at the mean radial velocity of the cluster. The other populations, including the over 100 classified dwarf ellipticals in Virgo with radial velocities, have much broader, and non-Gaussian, velocity distributions $\left(\sigma \sim 700 \mathrm{~km} \mathrm{~s}^{-1}\right)$, all with velocity dispersion ratios with the ellipticals consistent with their being accreted (e.g., Conselice et al. 2001). There is also significant sub-structure within these velocity distributions for dwarfs, unlike the case for the giant ellipticals.

Stellar Populations: Currently, we know with some certainty that dwarf galaxies have either young/metal rich or old/metal poor stellar populations (e.g., Poggianti et al. 2001). This is further seen in complete color-magnitude diagrams in nearby rich clusters, such as Perseus, down to $\mathrm{M}_{\mathrm{B}}=-12$ (Conselice 

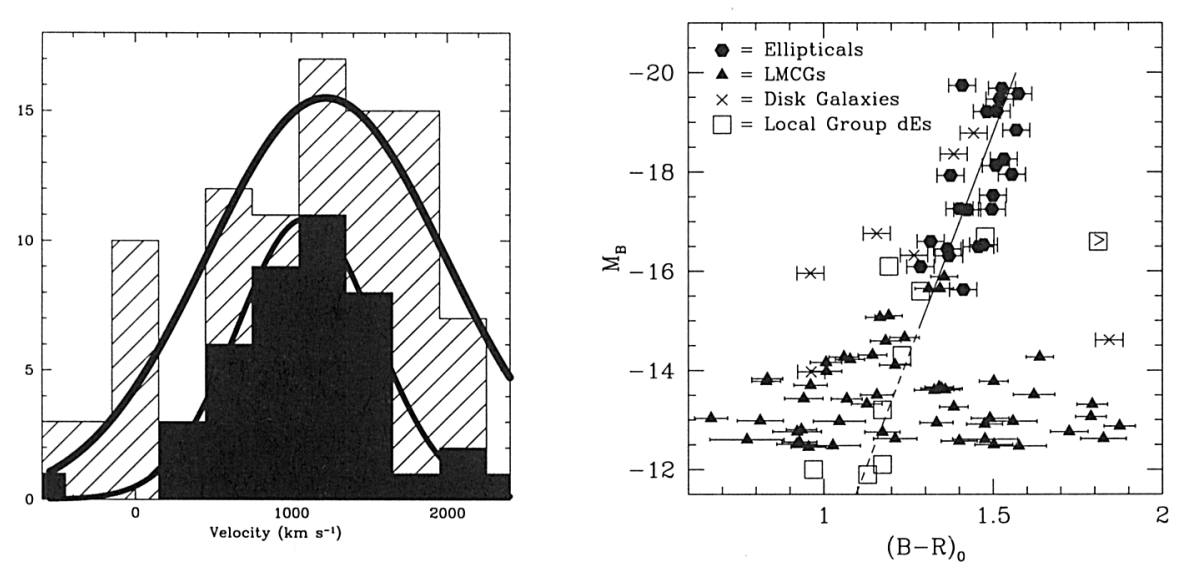

Figure 1. (a) Velocity histograms for giant ellipticals (solid) and dwarf ellipticals (shaded) in the Virgo cluster (Conselice et al. 2001) (b) Color magnitude diagram for galaxies in the Perseus cluster, demonstrating the large color scatter for systems with $\mathrm{M}_{\mathrm{B}}>-15$. The solid boxes are where Local Group dEs/dSphs would fit on this plot. Dwarf ellipticals are labeled as low mass cluster galaxies (LMCGs).

et al. 2002, 2003a). Fainter dwarfs also tend to be even more heterogeneous, with a large scatter from the color-magnitude relationship (CMR) (Conselice et al. 2003a; Rakos et al. 2001) (Figure 1b). This trend is found in several nearby clusters, including Fornax, Coma and Perseus, and can be explained by different dwarfs having mixtures of ages and metallicities (e.g., Poggianti et al. 2001; Rakos et al. 2001; Conselice et al. 2003a). Stromgren and broad-band photometry reveal that the redder dwarfs are metal enriched systems (Figure 2). Internal Kinematics: One key observational result, predicted in the simulations of Moore et al. (1998), is that a dwarf elliptical which has been transformed from a spiral should reveal some rotation. Using 8-10 meter class telescopes, the evidence is ambiguous with some dEs showing rotation (Pedraz et al. 2002), while others clearly do not (Geha et al. 2003). There is no obvious difference between these two populations in terms of morphology or chemical abundances (Geha et al. 2003), although these samples are still very small. The central velocity dispersions of these systems is also quite low, indicating that dark matter does not dominate, at least in their centers.

\section{Dwarf Galaxy Origins}

Any successful theory of dwarf galaxies, particularly dwarf ellipticals, must therefore explain the following properties: overdense in relation to massive galaxies in rich environments, mix of stellar populations, ability to survive in dense environments, diffuse spatial and velocity structure, and mixtures of rotation. This theory must also explain why fainter dwarfs are more heterogeneous than brighter ones. 

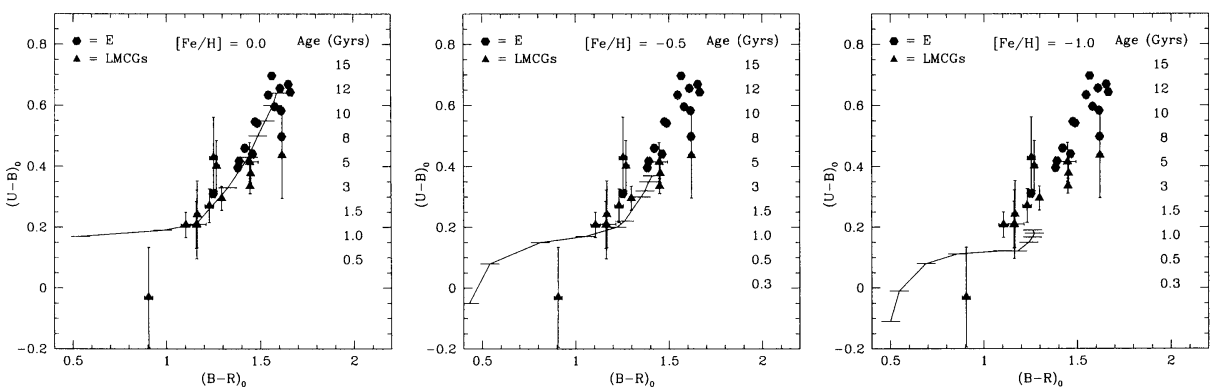

Figure 2. Three stellar synthesis modeled age tracks on a UBR colorcolor diagram at constant metallicities of solar, $[\mathrm{Fe} / \mathrm{H}]=-0.5$, and $[\mathrm{Fe} / \mathrm{H}]=-1$. The age range is $0.3 \mathrm{Gyrs}$ to $15 \mathrm{Gyrs}$ for the $[\mathrm{Fe} / \mathrm{H}]=-0.5$ and -1 models and 0.5 Gyrs to 15 Gyrs for the solar metallicity models. Dwarf ellipticals are labeled as low mass cluster galaxies (LMCGs).

One idea recently suggested by Tully et al. (2002) is that the dark matter halos of dwarfs are 'squelched' in lower density environments due to a large ultraviolet background after the universe was reionized. This explains the differences in $\alpha$ between different environments, but does not explain how within the same environment there is a great diversity in the dwarf population. It also does not easily explain why many dwarfs appear to have recently been accreted into clusters. In simple collapse + feedback scenarios (Dekel \& Silk 1986), dwarfs are formed when gas collapses and forms stars. These stars produce winds that expel gas from these systems, halting any future star formation. In this formation scenario dwarfs formed before the cluster ellipticals, or at least formed within groups that later merged to form clusters. Fainter dwarfs however, cannot all be born in groups which later accreted into clusters along with the massive galaxies, due to the high dwarf to giant galaxy ratio found in clusters (Conselice et al. 2001, 2003a). The above evidence suggests that simple low-mass galaxy formation scenarios can be safely ruled out for some dwarfs. One alternative idea is that some modern dwarfs formed after the cluster itself was in place by collapsing out of enriched intracluster gas. Another is that the intracluster medium (ICM) is able to retain enriched gas that in the Dekel and Silk (1986) paradigm would be ejected by feedback, but remains due to the confinement pressure of the ICM (Babul \& Rees 1992). This scenario would explain the higher metallicities of some of the fainter dwarfs.

An alternative scenario, now gaining in popularity, is that dwarfs form in clusters through a tidal origin. Two main possibilities for this are tidal dwarfs (Duc \& Mirabel 1994), and as the remnants of stripped disks or dwarf irregulars (Conselice et al. 2003a). The velocity and spatial distributions of dwarfs suggests that they were likely accreted into clusters during the last few Gyrs (Conselice et al. 2001). This, combined with the high metallicities of these cluster dwarfs, and the fact that their stellar populations are fundamentally different than field dwarfs (e.g., Conselice et al. 2003a; Figure 1b) suggests that the cluster environment has morphologically transformed, or stripped, accreted 

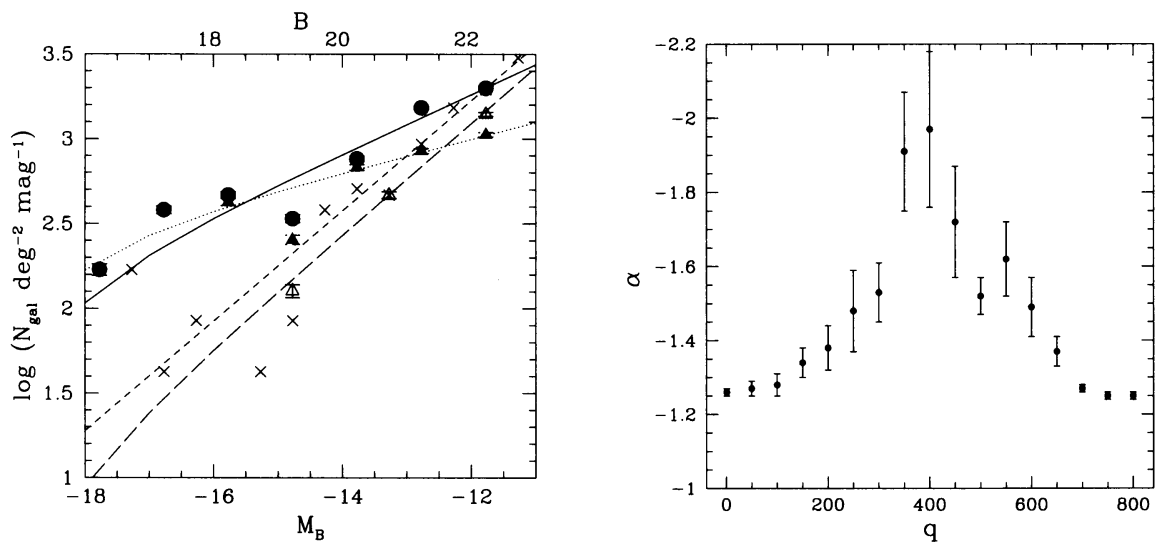

Figure 3. The Perseus cluster luminosity function plotted in various ways down to $M_{B}=-11$. The solid round points, and fitted solid line, is the total luminosity function of the central region of Perseus. The luminosity function for dEs redder and bluer than the CMR prediction are plotted as open and solid triangles and long dashed and dotted lines. The crosses mark the density of background galaxies. b: Modeled cluster luminosity function slope, $\alpha$, as a function of the number of high-speed maximum interactions $(\mathrm{q} \sim$ time $)$ cluster galaxies undergo during evolution in a Perseus like cluster (see Conselice 2002).

galaxy material into dwarfs. There is evidence for this process currently occurring in nearby clusters (e.g., Conselice \& Gallagher 1999). If dEs form from infall spirals then there should also be systems now being transformed which appear morphologically as dEs, but retain some of the gas left over from their precursor. These systems would only exist in the outer parts of clusters, as any that venture towards the core will be rapidly stripped of material. Very deep Arecibo observations of Virgo dEs reveals that $\sim 15 \%$ of a sample of 56 Virgo dEs have HI detections, all of which are located outside the core of the cluster (Conselice et al. 2003). Other detailed morphological investigations of nearby cluster dwarf ellipticals reveal that they come in a wide diversity of structures, some with tidal features, and others with apparent spiral structures (e.g., De Rijcke et al. 2003; Barazza et al. 2003; Graham et al. 2003).

\section{Implications of Dwarf Recycling}

Environment likely plays a role in all aspects of galaxy formation and evolution. If indeed these objects were formed after the cluster, due to a tidal process, there are several features of clusters that this can possibly explain. The first is that the LF of clusters will change after these lower mass galaxies form. The LF of galaxies in the Perseus cluster becomes flatter, with a similar faint end slope $(\alpha)$ as the field, after removing these red galaxies (Figure 3).

If these systems originate from tidally disturbed galaxies then the amount of light liberated is enough to account for all intracluster light (Conselice et al. 
2003a). For example, the total luminosity of intracluster light within $2^{\circ}$ of M87 is $2 \times 10^{11} \mathrm{~L}_{\odot}$. If this material originates from tidally stripped objects whose remnants are dwarfs, we can compute how much material on average each must have lost. There are $170 \mathrm{dEs}, 148 \mathrm{dE}, \mathrm{Ns}$, and $14 \mathrm{~S} 0$ galaxies within this radius in Virgo. On average, if all the $\mathrm{dE}$ and $\mathrm{dE}, \mathrm{N}$ galaxies are remnants of stripped galaxies then $0.6 \times 10^{9} \mathrm{~L}_{\odot}$ was lost by each object. If we consider only $\mathrm{dE}$ galaxies as remnants of this process then $1.1 \times 10^{9} \mathrm{~L}_{\odot}$ was lost by each $\mathrm{dE}$. This is enough material to suggest that each dwarf might have been a moderate-sized disk galaxy in the past.

Acknowledgments. I acknowledge my collaborators Jay Gallagher, Rosie Wyse and Karen O'Neil for their participation in the projects described here. I also thank the organizers of this symposium for creating an interesting meeting, and for their patience and support.

\section{References}

Babul, A., \& Rees, M.J. 1992, MNRAS, 255, 346

Barazza, F.D., Binggeli, B., \& Jerjen, H. 2003, A\&A, 407, 121

Conselice, C.J., \& Gallagher, J.S. 1999, AJ, 117, 75

Conselice, C.J., Gallagher, J.S., \& Wyse, R.F.G. 2001, ApJ, 559, 791

Conselice, C.J., Gallagher, J.S., \& Wyse, R.F.G. 2002, AJ, 123, 2246

Conselice, C.J., Gallagher, J.S., \& Wyse, R.F.G. 2003a, AJ, 125, 66

Conselice, C.J., et al. 2003b, ApJ, 591, 167

Conselice, C.J. 2002, ApJ, 573, 5L

De Rijcke, S., et al. 2003, A\&A, 400, 119

Dekel, A., \& Silk, J. 1986, ApJ, 303, 39

Duc, P.-A., \& Mirabel, I.F. 1994, A\&A, 289, 83

Ferguson, H.C., \& Binggeli, B. 1994, A\&ARv, 6, 67

Geha, M., Guhathakurta, P., van der Marel, R.P. 2003, AJ, 126, 1794

Graham, A.W., Jerjen, H., \& Guzman, R. 2003, AJ, 126, 1787

Kleyna, J., et al. 2002, MNRAS, 330, 792

Moore, B. et al. 1998, ApJ, 495, 139

Pedraz, S. et al. 2002, MNRAS, 332, 59L

Poggianti, B.M., et al. 2001, ApJ, 562, 689

Rakos, K., et al. 2001, AJ, 121, 1974

Sabatini, S., et al. 2003, MNRAS, 341, 981

Sandage, A., et al. 1985, AJ, 90, 1759

Trentham, N., et al. 2002, ApJ, 569, 573

Tully, R.B., et al. 2002, ApJ, 569, 573

van den Bergh, S. 2000, The Galaxies of the Local Group, Cambridge 